\title{
A cool liquid that does not freeze
}

A simulation study of a model that mimics certain colloidal particles reveals a surprising low-temperature triumph of entropy, whereby the liquid state persists down to zero temperature.

\section{Jeppe C. Dyre}

$\mathrm{n}$ classical physics there is no such thing as a thermodynamically stable liquid phase at $0 \mathrm{~K}$, as any liquid will freeze into a crystalline solid at sufficiently low temperatures ${ }^{1-4}$. In the real world the only exception to this is helium, for which quantum fluctuations stabilize the liquid state down to absolute zero. Liquids freeze upon cooling because energy eventually dominates over entropy, so at adequately low temperatures the crystalline state - which minimizes its energy by carefully packing the molecules - has a lower free energy than the liquid state.

The simulation study presented by Smallenburg and Sciortino 5 in Nature Physics now disproves this conventional wisdom by providing a concrete and realistic counterexample. They calculate the thermodynamic phase diagram of a system of colloidal particles with a valence of four. Their model, which is an extension of the Kern-Frenkel model ${ }^{6}$, consists of identical spherical particles with four circular attractive patches on their surfaces. Two particles are bonded with a fixed energy whenever their centre-of-mass distance is smaller than the interaction range and the vector connecting the particles passes through a patch on both particles (Fig. 1).

For small opening angles of the patches the phase diagram is fairly standard. Depending on density, three crystalline phases are found (diamond, body-centred cubic, face-centred cubic and mixtures of these). However, even at opening angles below $45^{\circ}$ a thermodynamically stable liquid state appears at zero temperature for a sizable range of densities. This is a great surprise, but fortunately the authors provide a simple explanation for the stability of a liquid phase even at the lowest temperatures.

For large opening angles there is great flexibility, allowing for a large number of fully bonded states. Most of these states are disordered, of course. As all fully bonded states have the same (low) energy, the minimum free energy is determined by maximizing the entropy. This quantity has two contributions: the configurational entropy that counts the number of different packings - zero for crystals - and the vibrational entropy, which is the logarithm

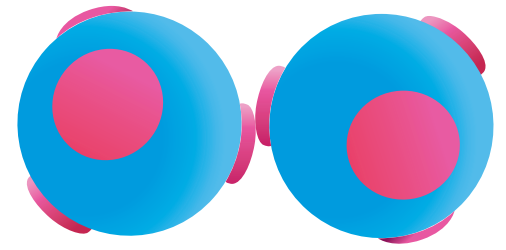

b

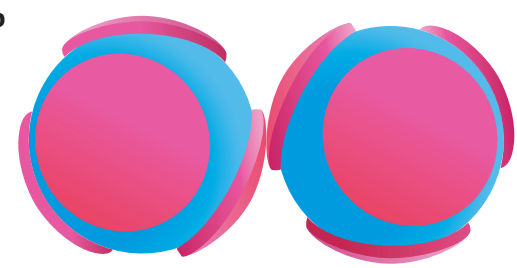

Figure 1 | Patchy colloids of the SmallenburgSciortino model. Each spherical particle has four 'patches' (red), which are areas for bonding to a nearby neighbouring particle. a, For particles with small patches the system crystallizes upon cooling. b, Large patches offer great flexibility, and thus many disordered 'fully bonded' states are possible, where each particle is bonded to four neighbours and the energy is consequently minimal. The entropy of these states is larger than that of the crystal. In this way the model's large binding angles and small valence (four in this case) give rise to a thermodynamically stable, fully bonded liquid phase.

of the vibrational phase-space volume of a single configuration. Because the crystalline state is carefully packed, at any given density this state has higher vibrational entropy than a typical liquid configuration. For sufficiently large opening angles, however, the liquid's vibrational entropy is close to the crystal's, and the total liquid entropy (taking into account the multitude of disordered packings) ends up being larger than that of the crystalline state. Thus the liquid is thermodynamically stable, even at the lowest temperatures.

What are the chances of realizing a liquid that never freezes? Smallenburg and Sciortino mention that their model mimics, for instance, systems of colloidal particles functionalized with four long DNA strands ${ }^{7}$. The results should also apply to more general flexible-bond models, however, and it seems likely that we shall soon see examples of the fully bonded disordered matter proposed in their study. The authors do note that any real system will eventually freeze, as forces are continuous and there is always some energetic contribution to the free energy. This merely introduces a very low freezing temperature, far below those of the higher-density freezing transitions of the system, and this does not make their basic observation less interesting.

The demonstration of a low-temperature liquid phase illustrates the diversity of complex liquids. This stands in sharp contrast to the regularities of simple liquids - for example, the famous Lennard-Jones model ${ }^{8}$ which, in the real world, are realized as most or all van der Waals and metallic liquids. Covalently bonded or hydrogen-bonded liquids like silica, water or alcohols - and now the Smallenburg-Sciortino model are much more spectacular, due to their directional bonding.

Another interesting implication of the investigation relates to the Kauzmann paradox. In 1948 Kauzmann noticed that the entropy of a supercooled liquid always extrapolates to zero well above zero temperature ${ }^{10}$, implying a negative liquid entropy at low temperatures. This is forbidden by the laws of thermodynamics. In practice, the glass transition intervenes, at which point the metastable liquid freezes into a glass. The paradox comes from the fact that the glass transition is not a genuine phase transition, but a dynamic falling-out-of-equilibrium. The temperature at which this happens is lower the slower the liquid is cooled, so sufficiently slow cooling should take the liquid to a state of negative entropy. Various resolutions have been presented ${ }^{11,12}$; perhaps the simplest idea is that a second-order phase transition takes place to a zero-entropy ideal glass state at the so-called Kauzmann temperature, at which the liquid's relaxation time becomes infinite ${ }^{13}$. The discovery of a liquid that does not freeze now presents a serious challenge to the Kauzmann paradox: here is a system that has no Kauzmann temperature and which, no matter how slowly it is cooled, forms a glass at a temperature where the liquid entropy is strictly positive. Thus the famous Kauzmann-Adam-Gibbs ${ }^{13,14}$ scenario of a second-order phase transition to a state of zero entropy and infinite relaxation 
time - supposed by many to underlie the observed glass transition - cannot apply universally.

Jeppe C. Dyre is in the Glass and Time group, Danish National Research Foundation Centre for Viscous Liquid Dynamics, IMFUFA, Department of Sciences, Roskilde University, PostBox 260, DK-4000 Roskilde, Denmark.

e-mail:dyre@ruc.dk
References

1. Atkins, P. W. Physical Chemistry 4 th edn (Oxford Univ. Press, 1990)

2. Debenedetti, P. G. Metastable Liquids (Princeton Univ. Press, 1996)

3. March, N. H. \& Tosi, M. P. Introduction to the Liquid State (World Scientific, 2002).

4. Barrat, J. L. \& Hansen, J. P. Basic Concepts for Simple and Complex Liquids (Cambridge Univ. Press, 2003).

5. Smallenburg, F. \& Sciortino, F. Nature Phys. 9, 554-558 (2013).

6. Kern, N. \& Frenkel, D. J. Chem. Phys. 118, 9882-9889 (2003).

7. Martinez-Veracoechea, F. J., Mladek, B. M., Tkachenko, A. V. \& Frenkel, D. Phys. Rev. Lett. 107, 045902 (2011).
8. Lennard-Jones, J. E. Proc. R. Soc. Lond. A 106, 441-462 (1924)

9. Ingebrigtsen, T. S., Schrøder, T. B. \& Dyre, J. C. Phys. Rev. X 2, 011011 (2012).

10. Kauzmann, W. Chem. Rev. 43, 219-256 (1948).

11. Angell, C. A. Science 267, 1924-1935 (1995).

12. Dyre, J. C., Hecksher, T. \& Niss, K. J. Non-Cryst. Solids 355, 624-627 (2009)

13. Lubchenko, V. \& Wolynes, P. G. Annu. Rev. Phys. Chem. 58, 235-266 (2007-)

14. Adam, G. \& Gibbs, J. H. J. Chem. Phys.

43, 139-146 (1965).

Published online: 4 August 2013

\section{QUANTUM MATERIALS}

\section{Shape resonances in superstripes}

The significance of 'stripes' in certain high-temperature superconductors has been hotly debated for decades. Now a consensus is emerging that there may, in fact, be two networks of different stripes in which shape resonances play a key role in the superconductivity.

\section{Antonio Bianconi}

$\mathrm{D}$ uring the last week of May 2013 two hundred selected experts on unconventional superconductivity, quantum electronics and multiferroics gathered together for the international meeting, Quantum in Complex Matter, the fourth conference in the 'Superstripes' series ${ }^{1}$. The site was the island of Ischia off the coast of Naples, Italy, which offers dramatic spots in a complex, unique landscape that has hosted Roman emperors Caligula and Tiberius, and writers such as Henrik Ibsen and Maxim Gorky.

This series of conferences started in 1992 with the Phase Separation workshop organized by K. A. Müller in Erice, Italy, followed by the Stripes conferences held in Rome and Erice from 1996 to 2008, when the Superstripes conferences began. The focus has been on the mechanisms of high-temperature superconductivity controlled by lattice effects, phase separation and multi-condensate superconductivity ${ }^{2}$. These issues are at the forefront of research today after having been considered a 'minority paradigm' for 25 years.

During Superstripes 2013 the idea emerged that controlling high-temperature superconductivity by fine tuning the lattice features is not chemistry, but low-energy physics. Whereas chemistry involves interactions in the range of $1-6 \mathrm{eV}$, here multiple electronic components are tuned around the Fermi energy, in the range of $20-100 \mathrm{meV}$. This is close to the physics of living matter, where atoms in motion control the low-energy physics of interactions in the range of 25-200 meV.

Many hot topics were discussed, ranging from chiral excitations to spin excitations, and from the deviation of the local structure from the average structure to phase separation in iron-based superconductors and oxide interfaces, quantum electronics, pressure effects, a

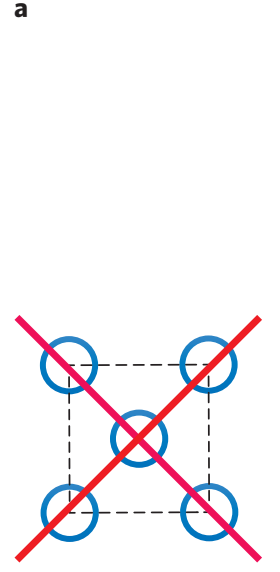

b
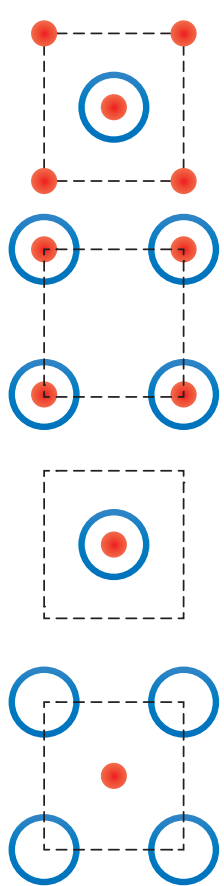

fast dynamics, and resonant and inelastic $\mathrm{X}$-ray diffraction studies. Here I focus on two subjects on which the community reached a solid consensus in hightemperature superconductivity.

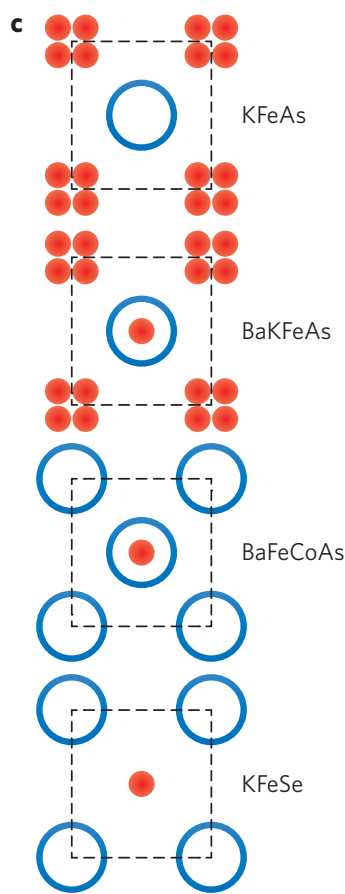

Figure 1 | Simplified Fermi surface topologies that favour multi-condensate superconductivity in iron-based superconductors. $\mathbf{a}$, The $s \pm$ model. $\mathbf{b}$, The shape resonance model. The blue circles are large Fermi surfaces and the red dots are small Fermi surface spots, which appear or disappear with small changes of the chemical potential at the centre or at the edges of the Brillouin zone (dashed line). c, Schematic of the Fermi surfaces detected by ARPES in different families of iron-based superconductors, as presented by Borisenko at Superstripes 20138,9. All families show new Fermi surface spots at optimum $T_{\mathrm{c}}$ that appear where the chemical potential is near a band edge, in agreement with the shape resonance model, whereas the nesting topology of the $s \pm$ model is never observed in the superconducting phase. 